\title{
The role of sensory nerve conduction study of the palmar cutaneous nerve in the diagnosis of carpal tunnel syndrome in patients with polyneuropathy
}

\author{
Tokçaer B. Ayse, Gögüs Feride*, Güllap Sümer**, Keles Isik*, Gökçe Mustafa**** \\ Gazi University, Department of Neurology, *Department of Physical Medicine and Rehabilitation, **Guven Hospital, Department of \\ Neurology, ${ }^{* *}$ Kirikkale University, Faculty of Medicine, Department of Physical Medicine and Rehabilitation, Ankara - Turkey, ${ }^{* * *}$ Sutcu \\ Imam University, Faculty of Medicine, Department of Neurology, Kahramanmaras, Turkey
}

Background: Conventional methods in the diagnosis of
carpal tunnel syndrome (CTS) in patients with
polyneuropathy (PNP) are insufficient. Aims: We suggest
that the comparison of the conduction of the median nerve
with that of the neighboring peripheral nerves may be more
beneficial in the diagnosis of entrapment neuropathy. Setting and Design: The median nerve sensory conduction in healthy volunteers, in cases of CTS, PNP cases without CTS and in cases of PNP in whom clinical findings point to CTS, were compared by palmar cutaneous nerve (PCN) sensory conduction. Materials and Methods: Comparative parameters were difference of $\mathrm{PCN}-1^{\text {st }}$ digits' nerve conduction velocities (NCV), PCN/1 $1^{\text {st }}$ digit NCVs ratio, difference of $5^{\text {th }}-2^{\text {nd }}$ digits' NCVs and $5^{\text {th }} / 2^{\text {nd }}$ digits NCVs ratio. Statistical Analysis: The statistical analysis was performed by the SPSS package for statistics. Student $t$ test and receiver operating characteristic were used. Results: Although the ratio of $\mathrm{PCN}-1^{\text {st }}$ digit did not differ significantly between the control group and the polyneuropathy group, there was a significant difference between CTS and PNP+CTS groups and the control group $(P<0.001$ and $P<0.001$, respectively). The ratio of $\mathrm{PCN}-1^{\text {st }}$ digit nerve conduction velocity was also significantly different between polyneuropathy and PNP+CTS groups $(P<0.001)$. Conclusion: To diagnose CTS on a background of polyneuropathy in mild cases in which sensory conduction is preserved, the ratio of sensory nerve conduction velocities of the palmar cutaneous nerve and the median nerve $1^{\text {st }}$ digit-wrist segment may be a criterion.

Key words: Carpal tunnel syndrome, nerve conduction study, palmar cutaneous
Carpal tunnel syndrome (CTS) is generally an idiopathic disorder, which occurs due to compression of the median nerve in the carpal tunnel. ${ }^{[1]}$ CTS is the most commonly seen entrapment neuropathy in the upper extremities. The diagnosis should be confirmed by electrodiagnostic methods in patients without objective sensory and motor deficits. ${ }^{[2]}$ When the conventional measures are normal, the study of comparative nerve conduction studies increases the sensitivity of the diagnosis by $9-11 \%{ }^{[3]}$

Polyneuropathy (PNP) and CTS are quite common in patients with diabetes mellitus. It is reported that the prevalence of CTS in patients with diabetic PNP is higher than in the general population and the risk of CTS is 2.2 times higher in diabetic women than in nondiabetic women and 2.5 times higher in diabetic than in nondiabetic men. $\cdot^{[4,5]}$

A dependable diagnosis of entrapment neuropathy in individuals with polyneuropathy is important for treatment indications. However, it is hard to claim whether the abnormalities in sensory nerve conduction of these cases are due to polyneuropathy or entrapment neuropathy. ${ }^{[2]}$

For the diagnosis of CTS on a background of polyneuropathy, it seems more dependable to rely on the comparative values of median nerve sensory conduction to different nerves of the same extremity, rather than the findings of motor and sensory conduction of the median nerve. In order to test our hypothesis, we compared the sensory nerve conduction velocity of the palmar cutaneous nerve that is the sensory branch of the median nerve, which does not pass through the carpal tunnel, to that of the $1^{\text {st }}$ digital branch of the median nerve.

Poster presentation at $\mathrm{XI}^{\text {th }}$ International Congress of EMG and Clinical Neurophysiology,

7-11 September 1999, Prague, Czech Republic 


\section{Materials and Methods}

\section{Subjects}

In this study, we examined patients who were referred to our electrophysiology laboratory to determine their nerve conduction studies. Clinical diagnosis of patients who came from the neurology or orthopedics departments was CTS. Other patients who were referred from the endocrinology department had noninsulin-dependent diabetes mellitus.

We evaluated four groups in this study. Group 1 was composed of patients with CTS who were diagnosed by electrophysiological methods, Group 2 was composed of patients who were diagnosed to have PNP by electrophysiological methods and who lacked findings of entrapment neuropathy, Group 3 were composed of cases of PNP with clinical symptoms of CTS and Group 4 was composed of healthy people with normal peripheral nerve conduction studies. Forty-one extremities of 26 cases from Group 1, 32 extremities out of 22 cases of PNP from Group 2 and that 32 extremities out of 24 cases from Group 3 with PNP and CTS were included in the study. The data from 66 extremities of 33 healthy volunteers, who comprised the control group, were evaluated.

\section{Testing methods}

Electrodiagnostic tests were studied by three investigators (SG, IK, MG) who were blind to the clinical diagnosis. The nerve conduction studies (NCS) of both sural nerves, peroneal and popliteal nerves in one of the lower extremities, bilateral median, ulnar and the palmar cutaneous nerves in the upper extremities were examined in all subjects. Polyneuropathy was diagnosed when the rate of abnormal findings was more than $50 \%$ in the examined nerves.

Electrodiagnostic studies were done by the Dantec Counter Point device at room temperature $26^{\circ} \mathrm{C}$. Skin temperatures of our cases ranged from $31^{\circ}-33^{\circ} \mathrm{C}$. Nerve conduction studies were performed by conventional methods in all nerves by using superficial stimulators and recording electrodes. The distal motor latencies were measured by stimulating the median nerve $5 \mathrm{~cm}$ proximal to the abductor pollicis brevis muscle (ABP) and by stimulating the ulnar nerve $5 \mathrm{~cm}$ proximal to the abductor digiti quinti muscle (ADQ). The $1^{\text {st }}, 2^{\text {nd }}, 3^{\text {rd }}$ digit-wrist (D-W) and palmar-wrist (P-W) segments of the median nerve were examined for sensory nerve conduction study, the wrist-elbow (W-E) segment was examined for mixed nerve conduction study and the fifth $\mathrm{D}-\mathrm{W}$ segment of the ulnar nerve was examined for sensory nerve conduction study by the orthodromic method. The conduction of the palmar cutaneous nerve (PCN) was examined by the orthodromic method as well. The PCN was stimulated from the thenar region by a superficial electrode. The sensory action potential was recorded $10 \mathrm{~cm}$ proximal to the point of stimulation [Figure 1]. The stimulation

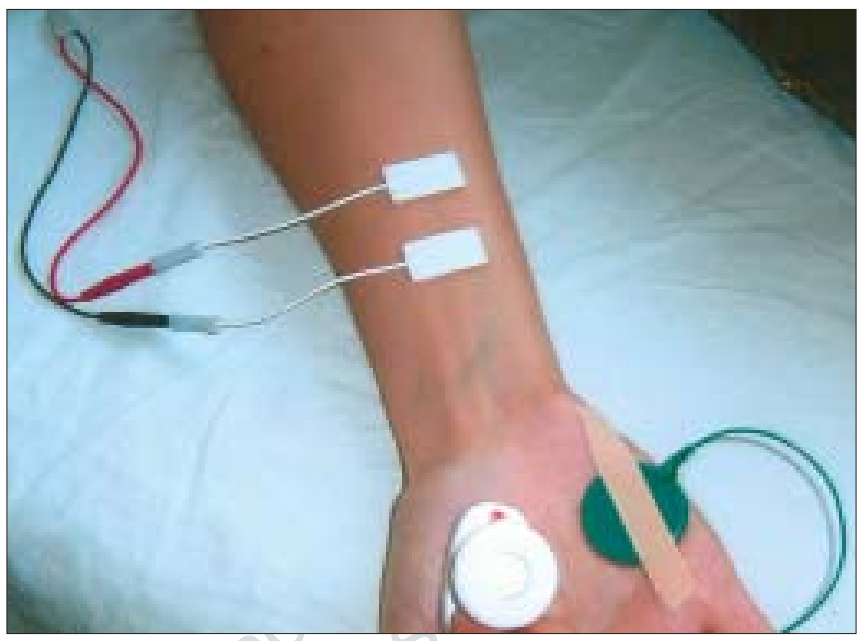

Figure 1: The method of recording of palmar cutaneous nerve sensory conduction

consisted of rectangular waves of $1 \mathrm{~Hz}$ frequency and 0.2 msec duration. The filter range was $20-2000 \mathrm{~Hz}$, the sweeping speed of the oscilloscope was $2 \mathrm{~ms} /$ division and the sensitivity was $10 \mu \mathrm{V} /$ division. High-intensity stimuli were avoided in order to prevent the stimuli from being spread to the thumb and to the region of the radial nerve. The latency measurement was made from the peak point of the compound sensory nerve action potential (SNAP). Comparative parameters were calculated according to the formula presented below:

PCN $-1^{\text {st }}$ difference $(\mathrm{m} / \mathrm{sec})=$ PCN NCV $-1^{\text {st }} \mathrm{D}-\mathrm{W}$ NCV (n. medianus)

$\mathrm{PCN} / 1^{\text {st }}$ ratio $=\frac{\text { PCN NCV }}{1^{\text {st }} \mathrm{D}-\mathrm{W} \text { NCV (n. medianus) }}$

$5^{\text {th }}-2^{\text {nd }}$ difference $=5^{\text {th }}$ digit-wrist NCV (n. ulnaris) $-2^{\text {nd }}$ D-W NCV (n. medianus)

$5^{\text {th }} / 2^{\text {nd }}$ ratio $=\frac{5^{\text {th }} \mathrm{D}-\mathrm{W} \text { NCV (n. ulnaris) }}{2^{\text {nd }} \mathrm{D}-\mathrm{W} \text { NCV }(\text { n. medianus })}$

\section{Data analysis}

The statistical analysis was performed by the SPSS package for statistics. The data from the control and patient groups were compared by the Student's t test. The $P$ value, two degree of freedom and $95 \%$ confidence interval, below 0.05 was considered statistically significant. Receiver operating characteristic (ROC) curves were done to determine the diagnostic sensitivity and specifity of comparative parameters.

\section{Results}

Table 1 presents the age, sex distributions, nerve conduction studies' (NCS) findings and the comparative NCS' findings of the control and patient groups. Nerve conduction velocities of the first three D-W segments and the P-W segment of the median nerve were 
Table 1: Demographic features and ulnar nerve NCS finding and differences of sensory NCVs of PCN and median, ulnar and median nerves

\begin{tabular}{lcccc}
\hline & Control & CTS & PNP & PNP+CTS \\
Age & $43.6 \pm 12.2$ & $46.1 \pm 11.0$ & $42.9 \pm 11.6$ & $41.1 \pm 11.1$ \\
Female/male & $21 / 12$ & $25 / 1$ & $17 / 5$ & $18 / 6$ \\
Ulnar latency (ms) & 2.42 & 2.42 & $2.77^{*}$ & $2.89^{*}$ \\
$5^{\text {th }}$ digit-wrist (m/sec) & 46.1 & 45.1 & $36.4^{*}$ & $36.4^{*}$ \\
PCN-1 $^{\text {st }}$ difference & 3.73 & $13.11^{*}$ & $1.60^{\dagger}$ & $8.71^{*}$ \\
$5^{\text {th }} 2^{\text {nd }}$ difference & -1.54 & $7.90^{*}$ & $0.96^{ \pm}$ & $2.89^{*}$ \\
\hline
\end{tabular}

CTS: Carpal tunnel syndrome, PCN: Palmar cutaneous nerve, PNP: Polyneuropathy, ${ }^{\star} P<0.001,{ }^{\dagger} P<0.005,{ }^{ \pm} P<0.05$

statistically different between the control and patients' groups [Figure 2].

The NCV of the median W-E segment, NCV of the PCN and the NCV of the $5^{\text {th }} \mathrm{D}-\mathrm{W}$ segment were not significantly different in the CTS groups $(P>0.5$ and $P>0.05$, respectively) [Figure 2]. Because the PCN- ${ }^{\text {st }}$ and $5^{\text {th }}-2^{\text {nd }}$ digit NCV differences were variable over a considerable range among the groups, the ratios of the NCVs of these segments were considered as more reliable criteria. Although the PCN-1 ${ }^{\text {st }}$ digit ratio did not differ significantly between the control group and the polyneuropathy group $(P>0.05)$, there was a significant difference between the control group and the CTS and PNP+CTS groups $(P<0.001$ and $P<0.001$, respectively) [Figure 3].

The $\mathrm{PCN} / 1^{\text {st }}$ digit NCV ratio was significantly different between the PNP and PNP+CTS groups as well $(P<0.001)$. The ratio of $5^{\text {th }} / 2^{\text {nd }}$ digit NCV was numerically close but the difference was statistically significant between the PNP and control groups. $(P<0.05)$ [Figure 3].

The ratios of the PCN $/ 1^{\text {st }}$ digit NCV and $5^{\text {th }} / 2^{\text {nd }}$ digit NCV were also significantly different between the CTS and PNP+CTS groups $(P<0.05$ and $P<0.005$, respectively) [Figure 3].

The ratio of PCN $/ 1^{\text {st }}$ digit NCV was above two standard deviations from normal in 33 extremities (80.4\%) from

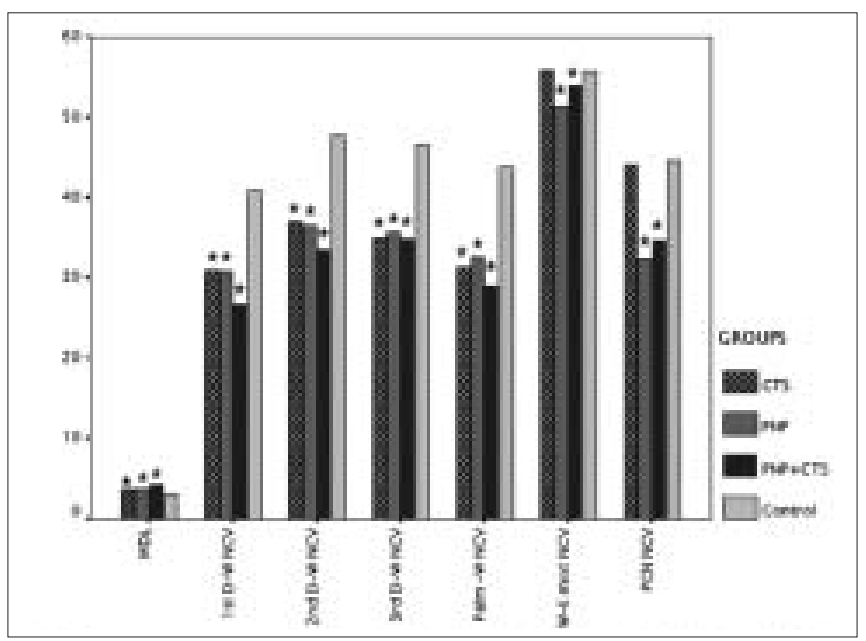

Figure 2: Median nerve conduction studies' findings in patients and control groups. ${ }^{\star} P<0.001$ the CTS group and in 29 extremities (90.6\%) from the PNP+CTS group. The $5^{\text {th }} / 2^{\text {nd }}$ digit NCV ratios were abnormal in the 22 extremities (53.6\%) of the CTS group and in five extremities (15.6\%) from the PNP+CTS group.

The ratio of PCN $/ 1^{\text {st }}$ digit NCV had sensitivity of $95 \%$ and specificity of $71 \%$. The sensitivity and specificity of $5^{\text {th }} / 2^{\text {nd }}$ digit NCV ratio was $92 \%$ and $59 \%$ respectively (Figure 4).

\section{Discussion}

In the present study, the sensory NCVs of the PCN and $5^{\text {th }} \mathrm{D}$-W segment of the ulnar nerve were not significantly different from the control group in CTS patients. In the PNP group, the difference in the sensory NCV of PCN and $1^{\text {st }} \mathrm{D}-\mathrm{W}$ segment and the difference in the sensory NCV of the $5^{\text {th }} \mathrm{D}-\mathrm{W}$ segment of the ulnar nerve and the $2^{\text {nd }} \mathrm{D}-\mathrm{W}$ segment of the median nerve were significantly lower than the control group. This situation was due to the slowness of the NCV in all nerves due to PNP.

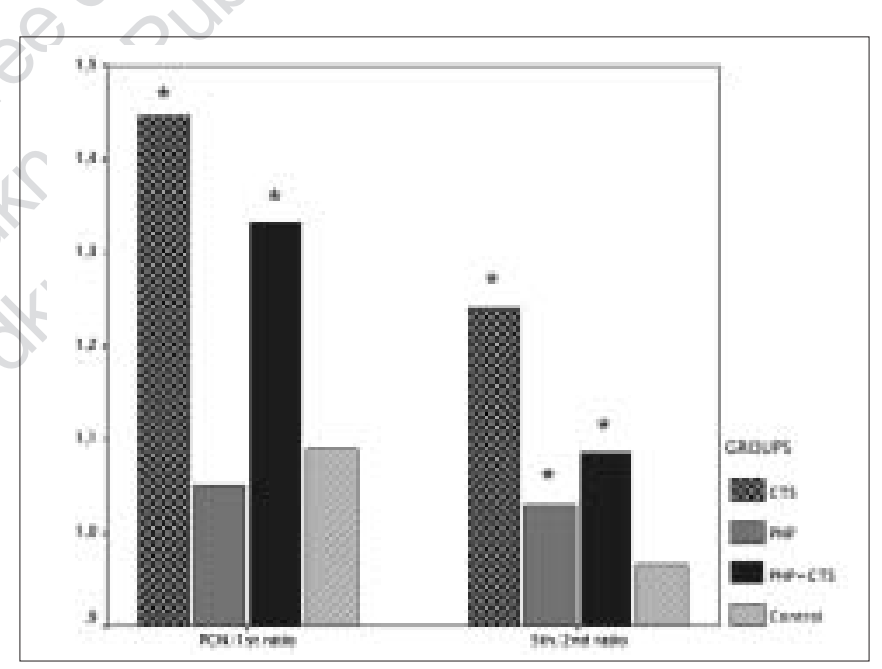

Figure 3: Comparisons of ratios of sensory nerve conduction velocities in patients and control groups. ${ }^{*} P<0.001$

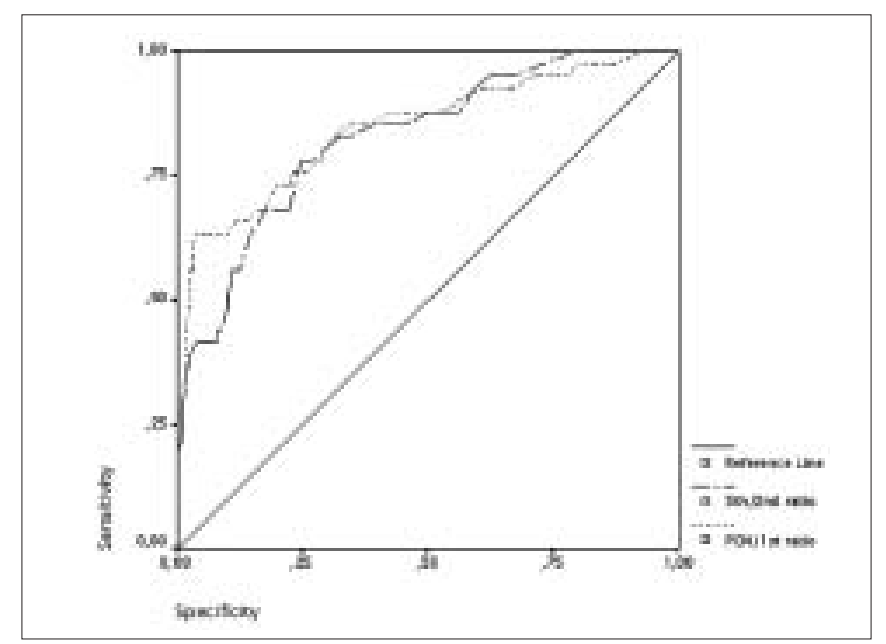

Figure 4: Receiver operating characteristic $(\mathrm{ROC})$ curves of comparative tests 
Therefore, the ratio, rather than the difference of sensory $\mathrm{NCV}$, is considered to be more reliable to diagnose CTS in PNP patients. The ratio of the PCN to the $1^{\text {st }}$ digit did not differ in control and PNP groups, whereas it was significantly higher in the CTS and CTS+PNP groups than in the control group.

Characteristic findings in the electrophysiological diagnosis of CTS are slowing in the sensory conduction in one or more branches of the median nerve and decrease in the amplitude of SNAP. In previous reports, the most sensitive electrophysiological finding of mild CTS was addressed as the slowing of sensory nerve conduction velocity in the $\mathrm{P}-\mathrm{W}$ and $1^{\text {st }} \mathrm{D}-\mathrm{W}$ segments. ${ }^{[1,6]}$

The American Association of Electrodiagnostic Medicine (AAEM) has concluded that median nerve sensory transmission was more sensitive than motor transmission in the diagnosis of CTS. The distal motor latency of the median nerve is not accepted as a standard for the diagnosis; the techniques that evaluate the sensory transmission between short distances and the comparison of the median nerve conduction to other nerve conductions on the same hand seem to be superior to the measurement of sensory nerve conduction in D-W segments, which are 13-14 cm long. The comparative studies eliminate the differences of conduction rates between individuals, because the patient may constitute his/her own control. ${ }^{[3,7,8]}$ Jackson et al detected abnormalities in the distal sensory latency differences of median-radial and median-ulnar nerves when the motor and sensory conduction studies were normal by conventional methods in cases of mild CTS. ${ }^{[9]}$

Another method in the diagnosis of CTS is to compare the sensory NCV of the PCN with the NCV of the digital branches of the median nerve. ${ }^{[10,11]}$

Because CTS and PNP cannot be differentiated with median nerve distal motor latency or the NCS of the nerve fibers that pass through the carpal tunnel, Vogt et al. measured the median distal motor latency from the second lumbrical muscle which progresses deeper in the tunnel rather than the APB muscle in cases of PNP. ${ }^{[2,4]}$ They recorded the ulnar nerve distal motor latency from the second interosseous muscle and used the difference in the diagnosis of CTS. By this method, the specificity of CTS diagnosis in PNP cases was 78\%, whereas the diagnostic specificity of the latency differences from abductor pollicis and abductor digiti quinti muscles was $43 \%$. This result suggested that recording from the lumbrical and interosseous muscles is more reliable. ${ }^{[2]} \mathrm{In}$ a study in which asymptomatic electrophysiological CTS was searched in diabetic patients without PNP, sensory NCV of the median and ulnar nerve D-W segments and the ratio of the terminal motor latencies were found to be significantly different. On the contrary, the ratios of motor and mixed NCV in the forearm segment were found similar to those of the control group. ${ }^{[2]}$ In a study in which median-ulnar comparative studies were used to determine the criteria of CTS on the basis of axonal polyneuropathy, the diagnostic value of the differences of the median and ulnar nerve distal motor latencies recorded from the lumbrical and interosseous muscles was found to be higher than the difference of sensory nerve latency differences. ${ }^{[13]}$

On the contrary, there is no clear evidence of the utility of electrodiagnostic testing in clinically definite CTS. It is reported that high-quality prospective studies should be designed to determine the predictive value of electrodiagnostic parameters in subclinic CTS. ${ }^{[14]}$

In our study, the ratio of sensory NCVs was the criterion because the sensory nerve action potentials could be recorded. Our cases were mild, in terms of both CTS and polyneuropathy. Because CTS does not affect the motor nerve transmission in early phases, comparison of sensory NCSs must be performed. On the other hand, it is clear that comparison of motor NCSs will be more beneficial in cases of severe CTS or polyneuropathy, due to the fact that SNAPs cannot be recorded. In our study, we compared the ulnar nerve and median $2^{\text {nd }} \mathrm{D}-\mathrm{W}$ segment as their lengths were similar. We suggest that the $2^{\text {nd }} \mathrm{D}-\mathrm{W}$ segment is affected later in CTS so the abnormality in the ratio of NCVs is mild.

\section{Conclusion}

We suggest that $\mathrm{PCN} / 1^{\text {st }} \mathrm{D}-\mathrm{W}$ NCV ratio is a reliable parameter for diagnosis of subclinic or mild CTS in cases with axonal polyneuropathy. Further studies may help to make interpretations on the sensitivity and specificity of this test in demyelinating polyneuropathies such as chronic inflammatory demyelinating polyradiculopathy.

\section{References}

1. Macdonell RA, Schwartz MS, Swash M. Carpal tunnel syndrome: Which finger should be tested? An analysis of sensory conduction in digital branches of the median nerve. Muscle Nerve 1990;13:6016.

2. Vogt T, Mika A, Thömke F, Hopf HC. Evaluation of carpal tunnel syndrome in patients with polyneuropathy. Muscle Nerve 1997;20:153-7.

3. Padua L, Lo Monaco M, Valente EM, Tonali PA. A useful electrophysiologic parameter for diagnosis of carpal tunnel syndrome. Muscle Nerve 1996;19:48-53.

4. Perkins BA, Olaleye D, Bril V. Carpal tunnel syndrome in patients with diabetic polyneuropathy. Diabetes Care 2002;25:565-9.

5. Müler-Felber W, Landgraf R, Reimers CD, Scheuer R, Wagner S, Nusser J, et al. High incidence of carpal tunnel syndrome in diabetic patients after combined pancreas and kidney transplantation. Acta Diabetol 1993;30:17-20.

6. Aydýn G, Keles I, Özbudak Demir S, Baysal AÝ. Sensitivity of median sensory nerve conduction tests in digital branches for the diagnosis of carpal tunnel syndrome. Am J Phys Med Rehabil 2004;83:17-21.

7. Jackson DA, Clifford JC. Electrodiagnosis of mild carpal tunnel syndrome. Arch Phys Med Rehabil 1989;70:199-204

8. Chang CW, Lien IN. Comparison of sensory nerve conduction in 
the palmar cutaneous branch of the median nevre: A new diagnostic method for carpal tunnel syndrome. Muscle Nevre 1991;14:11736 .

9. Imai T, Wada T, Matsumuto H. Entrapment neuropathy of the palmar cutaneous branch of the median nevre in carpal tunnel syndrome. Clin Neurophysiol 2004;115:2514-7.

10. Jablecki CK, Andary MT, Floeter MK, Miller RG, Quartly CA, Vennix MJ, et al. Second AAEM literature review of the usefulness of nerve conduction studies and needle electromyography for the evaluation of patients with carpal tunnel syndrome. Muscle Nerve 2002 .

11. American Association of Electrodiagnostic Medicine, American Academy of Neurology and American Academy of Physical Medicine and Rehabilitation. Practice parameter for electrodiagnostic studies in carpal tunnel syndrome: Summary statement. Muscle Nerve
$2002 ; 25: 918-22$.

12. Kim WK, Kwon S, Lee SH, Sunwoo IN. Asymptomatic electrophysiologic carpal tunnel syndrome in diabetics: Entrapment or polyneuropathy. Yonsei Med J 2000;41:123-7.

13. Ubogu EE, Benatar M. Electrodiagnostic criteria for carpal tunnel syndrome in axonal polyneuropathy. Muscle Nerve 2006;33:747 52 .

14. Jordan R, Carter T, Cummins C. A systematic review of the utility of electrodiagnostic testing in carpal tunnel syndrome. Br J Gen Pract 2002;52:670-3.

Accepted on 04-12-2006

Source of Support: Nil, Conflict of Interest: None declared. 